\title{
Review of: "Ethnobotanical Study On Plants Used To Dye Traditional Costumes By The Baiku Yao Nationality of China"
}

Yao Fu

Potential competing interests: The author(s) declared that no potential competing interests exist.

\section{Comments to Authors}

\section{General comments:}

This research documented detailed traditional knowledge on Dyeing traditional costumes of Baiku Yao people in China, the data presentation of the research is clear. However, the presentation of the discussion and conclusion part could be strengthened to make the argument more clear and strong. In addition, to protect the tradition knowledge of Baiku Yao people, issues on Access and Benefit Sharing on genetic resources and traditional knowledge (ABS) should be mentioned in the manuscript. Please also check attached PDF file for some detailed comments as well.

Specific comments:

\subsection{Statistical analyses}

According to the medicinal plant data presented in the manuscript, there is little meaning to use FIC index for data analysis. As the authors mentioned "FIC index is to deduce whether the informant's information was consistent when a specific plant was used to treat a particular category of ailments". The starting point for using FIC is the ailments and the purpose is to investigate most mentioned plants used for a particular category of ailments; I believe Baiku Yao people use more than 1 plant species for relieving cough. Use Report is well enough to represent the data, so I suggested deleting the FIC part in the methods and Results part.

\section{Results}

Please see the attached PDF file for details.

\section{Discussion}




\subsection{The diversity and uniqueness of traditional knowledge of dyeing Baiku Yao national clothing}

According to the title of this section, the authors mainly discussed two things: diversity and uniqueness of dyeing knowledge of Baiku Yao. But from a reader point of view, the presentation of the manuscript is not very clear. I felt that the third paragraph in this section mainly discussed about the uniqueness, while the second and the fourth paragraph were mainly talking about the diversity. It would be much better if the authors rearrange the paragraph and state the two points more clear.

\subsection{The potential value of dyeing plants used in Baiku Yao traditional clothing}

According to the data presented in this manuscript, the title of this section would be better to use "the potential value of dyeing plants and related traditional knowledge used in Baiku Yao traditional clothing"

To make the statements clear and strong, it is better to clearly point out what are the potential values, rather than discuss them species by species. According to the text, I think there could be four potential values: unique anti-dyeing knowledge (resin of A. vilmoriniana); later color fixing and brightness knowledge; reddish brown color knowledge; healthcare uses knowledge of dyeing plants. So, I suggest the authors could rearrange the statements.

\subsection{We should strengthen the protection of traditional knowledge of dyeing plants used in Baiku Yao traditional clothing}

Please see the attached PDF file for details.

\section{Conclusion}

To make the conclusion stronger, important implications of traditional knowledge on cloth dyeing of Baiku Yao people should be summarized in Conclusion part; current conclusion is too general and could be used in any other research study dye traditional knowledge. 